\title{
Use of a-SiC:H Semiconductor-Based Transducer for Glucose Sensing through FRET Analysis
}

\author{
Paula Louro ${ }^{1,2}$, Vitor Silva ${ }^{1,2}$, Manuel A. Vieira ${ }^{1,2}$, \\ A. Karmali ${ }^{4}$, and Manuela Vieira ${ }^{1,2,3}$ \\ ${ }^{1}$ Electronics Telecommunications and Computer Dept, ISEL, Lisbon, Portugal \\ ${ }^{2}$ CTS-UNINOVA, Quinta da Torre, 2829-516, Caparica, Portugal \\ ${ }^{3}$ DEE-FCT-UNL, Quinta da Torre, 2829-516, Caparica, Portugal \\ ${ }^{4}$ CIEB-ISEL, R. Conselheiro Emidio Navarro, 1, Lisbon, Portugal
}

\begin{abstract}
Glucose sensing is an issue with great interest in medical and biological applications. One possible approach to glucose detection takes advantage of measuring changes in fluorescence resonance energy transfer (FRET) between a fluorescent donor and an acceptor within a protein which undergoes glucose-induced changes in conformation. This demands the detection of fluorescent signals in the visible spectrum. In this paper we analyzed the emission spectrum obtained from fluorescent labels attached to a protein which changes its conformation in the presence of glucose using a commercial spectrofluorometer. Different glucose nanosensors were used to measure the output spectra with fluorescent signals located at the cyan and yellow bands of the spectrum. A new device is presented based on multilayered $\mathrm{a}-\mathrm{SiC}: \mathrm{H}$ heterostructures to detect identical transient visible signals. The transducer consists of a p-i'(a-SiC:H)-n/p-i(a-Si:H)-n heterostructure optimized for the detection of the fluorescence resonance energy transfer between fluorophores with excitation in the violet $(400 \mathrm{~nm})$ and emissions in the cyan $(470 \mathrm{~nm})$ and yellow $(588 \mathrm{~nm})$ range of the spectrum. Results show that the device photocurrent signal measured under reverse bias and using appropriate steady state optical bias, allows the separate detection of the cyan and yellow fluorescence signals.presented.
\end{abstract}

Keywords: Glucose sensing, FRET, nanosensors, fluorescence proteins, a$\mathrm{SiC}: \mathrm{H}$ photodiodes, multilayered heterostructures, optical sensors.

\section{$1 \quad$ Introduction}

There is a great scientific and medical interest to develop suitable technology for continuous in vivo glucose monitoring in patients with diabetes mellitus. There are several problems with existing devices based on electrochemistry which have encouraged alternative approaches to glucose sensing in recent years. An approach based on fluorescence intensity and lifetime, has special advantages, including sensitivity and the potential for non-invasive measurement when near infrared light is used. Such techniques include measuring changes in fluorescence resonance energy 
transfer (FRET) between a fluorescent donor and an acceptor within a protein which undergoes glucose-induced changes in conformation [1]. First fluorescence excitation is performed at the donor wavelength. As the protein changes its conformation the donor and acceptor fluorophores, attached to the protein, change their distance. When the distance between fluorophores is reduced the donor transfers energy to the acceptor through nonradiative dipole-dipole coupling. The energy transfer causes on one hand a decrease in intensity at the donor emission wavelength and on the other an increase in intensity at the acceptor emission wavelength. Such variation in the emission spectrum can be used to detect and measure the concentration of glucose in the sample.

In this paper we use a-SiC:H optical devices to detect the cyan and yellow emission signals of fluorescent proteins. The transducer consists of two heterostructures based on a-SiC:H/a-Si:H optimized for the detection of the fluorescence emissions at wavelengths $470 \mathrm{~nm}$ (cyan) and $588 \mathrm{~nm}$ (yellow) [2].

\section{Contribution to Internet of Things}

Internet of Things refers to the concept of expanding the communication among things. This will bring the ability of objects behaviour to change due to what they access through the Internet. In a FRET based sensor with a wireless system, a warning or emergency message could be generated and sent in a multicast network. This same message would be received by the nearest hospital and by a patient transportation system and also by the patient's doctor cell phone. Another scenario would be a feedback response based on the transmitted values which would tell the patient what to do.

Devices based on new materials for glucose sensing are an important contribution to the future development of the Internet of Things.

\section{Materials and Methods}

The following recombinant plasmids were purchased from Addgene (USA): 13561 pRSET FLIPglu-2uDelta13 and 13660 pRSET FLIPglu-3.2mDelta13. Luria Bertani culture medium, iminodiacetic acid, epichlrohydrin, butanodioldiglycidyl ether and ampicillin were obtained from Sigma Chemical Company (USA) and Sepharose 6B, Sepharose CL 4B, and Sepharose CL 6B were purchased from GE HealthCare (Sweden).

All cell-free extracts of recombinant E. coli strains were assayed for protein by using the Coomassie blue dye binding method.

The recombinant plasmids were used to transform E. coli DH5 $\alpha$ strain which was grown overnight in Luria Bertani (LB) culture medium containing $100 \mu \mathrm{g} / \mathrm{ml}$ of ampicillin at $37^{\circ} \mathrm{C}$ and $180 \mathrm{rpm}$ as described previously [1]. The cultures were harvested by centrifugation at $10.000 \mathrm{rpm}$ for $1 \mathrm{~min}$. at $4^{\circ} \mathrm{C}$ and the cells were ressuspended in 2 volumes of $20 \mathrm{mM}$ phosphate buffer $\mathrm{pH} 7.0$ containing $1 \mathrm{mM}$ benzamidine and sonicated five times for $30 \mathrm{~s}$ at $160 \mathrm{~W}$ with an interval of $5 \mathrm{~min}$. The 
cell-suspension was centrifuged at $20.000 \mathrm{rpm}$ for $1 \mathrm{~h}$ to remove cell debris and the supernatant was frozen at $-20^{\circ} \mathrm{C}$ for further studies. The cell-free extract was used as the source of glucose binding protein which was purified by immobilized metal affinity chromatography (IMAC) on immobilized copper chelates as described previously [3].

Fluorescence measurements were carried out in a Jasco FP-8300 spectrofluorometer equipped with the standard $10 \mathrm{~mm}$ cuvette holder.

Wavelength scans were performed on all glucose binding protein variants to determine excitation and emission peaks. Spectra were collected from 450-700 nm using the following configuration: bandwidth (excitation/emission) $-5 \mathrm{~nm}$; excitation wavelength - $433 \mathrm{~nm}$, data pitch $-0.2 \mathrm{~nm}$, scanning speed $-125 \mathrm{~nm} / \mathrm{min}$.

Unless otherwise stated, the fluorescent intensity per unit protein was determined with the samples diluted in $20 \mathrm{mM}$ sodium phosphate buffer $\mathrm{pH}$. 7.0 with excitation and emission set at $433 \mathrm{~nm}$ and 485 and $528 \mathrm{~nm}$, respectively. Glucose solutions were prepared in $20 \mathrm{mM}$ phosphate buffer $\mathrm{pH} 7.0$ containing $1 \mathrm{mM}$ benzamidine which were added to the cell-free extract containing the glucose binding protein variants to a final concentration of $100 \mathrm{mM}$ as follows: pRSET FLIPglu-2uDelta13 and pRSET FLIPglu-3.2mDelta13. Representative scans of the prepared glucose nanosensors are shown in Figure 1.
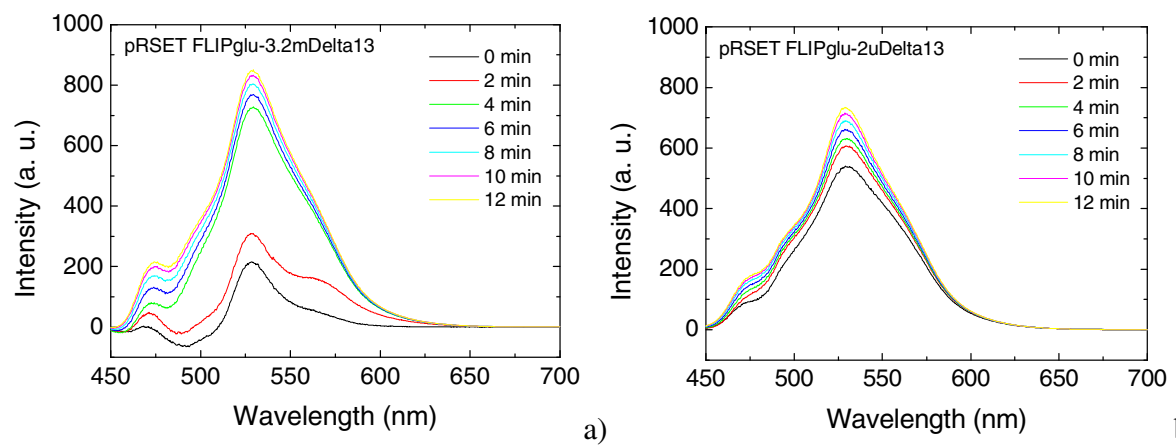

Fig. 1. Emission spectra for glucose nanosensors scanned during 12 minutes for: a) 13660 pRSET FLIPglu-3.2mDelta13 and b) 13561 pRSET FLIPglu-2uDelta13

In both spectra it is clear the presence of fluorescent emissions with peaks at the cyan and yellow regions. The observed spectrum changes along the reaction time are due to the induced conformation changes of the fluorescent proteins that stabilize after a few minutes. The correlation of these changes with time is probably related to the use of cellular extracts in our samples. The saturation curves are related to the glucose sensor concentration of the sample. Results show also that when the reaction is complete and saturation occurs the emission spectrum of both sensors exhibit similar trends. However, the cyan peak is more evident in glucose nanosensor pRSET FLIPglu-3.2mDelta13. 


\section{Device Configuration}

The device described herein works from 400 to $700 \mathrm{~nm}$ which makes it suitable for FRET applications using fluorophore operating in the visible spectrum. The device is a multilayered heterostructure based on a-Si:H and a-SiC:H. The configuration of the device includes two stacked p-i-n structures between two electrical and transparent contacts (Fig. 2). Both front (pin1) and back (pin2) structures act as optical filters confining, respectively, the short and the long optical carriers, while the intermediate wavelengths are absorbed across both [4,5].

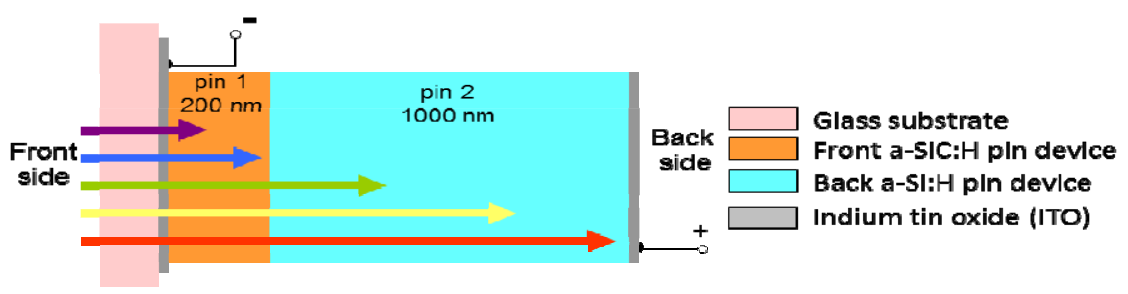

Fig. 2. Simplified schematic diagram of the structure of the device

The device was operated within the visible range using as optical signals to simulate the emitted fluorescent signals of the recombinant plasmids from E. coli strains (yellow and cyan) the modulated supplied by a cyan and a yellow LED with wavelengths of $470 \mathrm{~nm}$ and $588 \mathrm{~nm}$, respectively. Steady state optical bias of different wavelength from the front and back sides was also used for light soaking the device.

\subsection{Spectral Sensitivity}

Figure 3 displays the spectral photocurrent, measured along the visible spectrum, under reverse bias without and with optical light bias focusing the device from back and front sides.
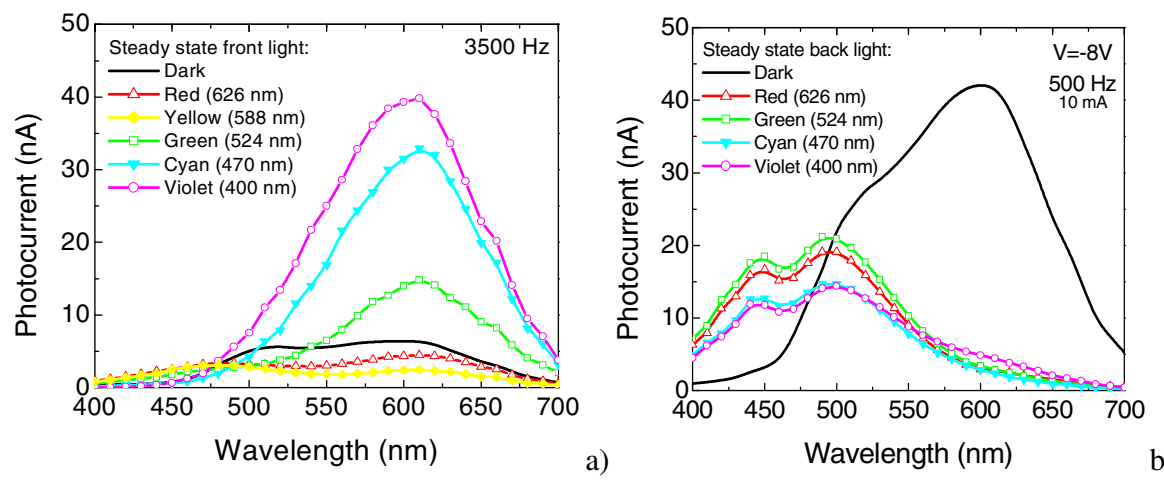

Fig. 3. Spectral photocurrent under dark conditions and using steady state light of different wavelengths by: a) front and b) back sides of the device 
Results of Fig. 3a) show that the use of steady state light bias from the front side, i.e., from the a-SiC:H structure, enhances the photocurrent in the range of wavelengths longer than $500 \mathrm{~nm}$ when using background light of short wavelength (400 nm, $470 \mathrm{~nm}$ and $524 \mathrm{~nm}$ ). For optical bias of longer wavelengths (588 nm and $626 \mathrm{~nm}$ ) the photocurrent signal is decreased. On the remaining part of the spectrum an opposite behavior is observed as the amplification effect occurs for longer light bias wavelengths and the reduction of the signal for shorter wavelengths. Thus, the use of a $400 \mathrm{~nm}$ light source as optical bias amplifies both cyan and yellow fluorescent. On the other hand, the optical biasing from the back side (a-Si:H) is not so much dependent on the background light wavelength (Fig. 3b). Generally, in the range of wavelengths shorter than $480 \mathrm{~nm}$ it is observed an increase of the signal for every background, and a decrease in the complementary range. The enhancement of the photocurrent signal using different optical bias for the background light result is an effective method for tuning the device sensitivity and detecting the emission fluorescent wavelengths.

\subsection{Transfer Function of Each Fluorescent Optical Signal}

The photocurrent signal obtained at reverse electric bias (-8 V) with (dark lines) and without background light (violet at $400 \mathrm{~nm}$ from the front side and cyan at $470 \mathrm{~nm}$ from the back side) is displayed in Fig. 4 for simulated fluorescent optical signals (cyan and yellow emissions, respectively, at $470 \mathrm{~nm}$ and $588 \mathrm{~nm}$ ).
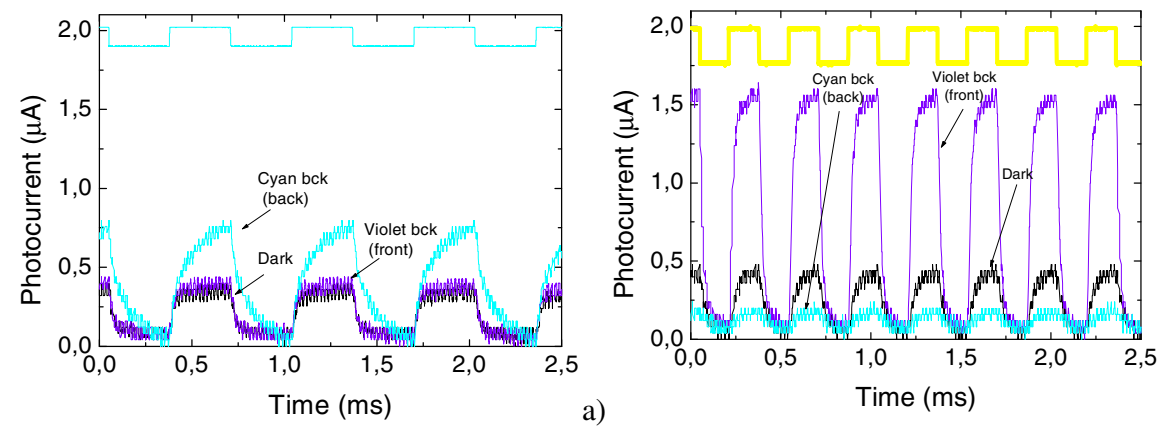

b)

Fig. 4. Photocurrent signal obtained at reverse bias without and under different background lights with the: a) cyan $(470 \mathrm{~nm})$ and b) yellow $(588 \mathrm{~nm})$ signals

As already indicated by the spectral photocurrent measurements of Fig. 4 the same wavelength is focusing on the device back side. The use of violet light from the front side has a negligible effect on this signal. For the yellow emission at $588 \mathrm{~nm}$ the effect of the background light is opposite. The violet steady state light from the front side causes a large amplification of the photocurrent (amplification factor about 4 times) and at the same time the cyan light from the back side reduces the signal by half. This amplification mechanism is useful for the determination of each fluorescent signal contribution to the whole output signal. With cyan background light from the 
back side the cyan fluorescent signal is enhanced and the yellow one reduced. On the other hand violet background from the front side will increase the photocurrent component due to the yellow signal.

\subsection{Transfer Function of a FRET Optical Signal}

Several time dependent wavelength combinations of both fluorescent pulsed input optical signals (at $470 \mathrm{~nm}$ and $588 \mathrm{~nm}$ ) were used to generate a multiplexed signal in the device. The output photocurrents, with and without optical background light are displayed in Figure 5. The reference level was assumed to be the signal when all the input optical signal channels are OFF. At the top of the figure, the individual optical signals are displayed to guide the eyes in relation to the different ON-OFF states. In both chromatic sequences it is clearly observed that the shape of the photocurrent signal measured under dark (black line) exhibits four threshold levels, each assigned to the correspondent optical conditions of the input signals. The ON-ON state corresponds to the maximum intensity of light bias, while the ON-OFF and OFF-ON to a lower intensity and the OFF-OFF to the dark conditions.
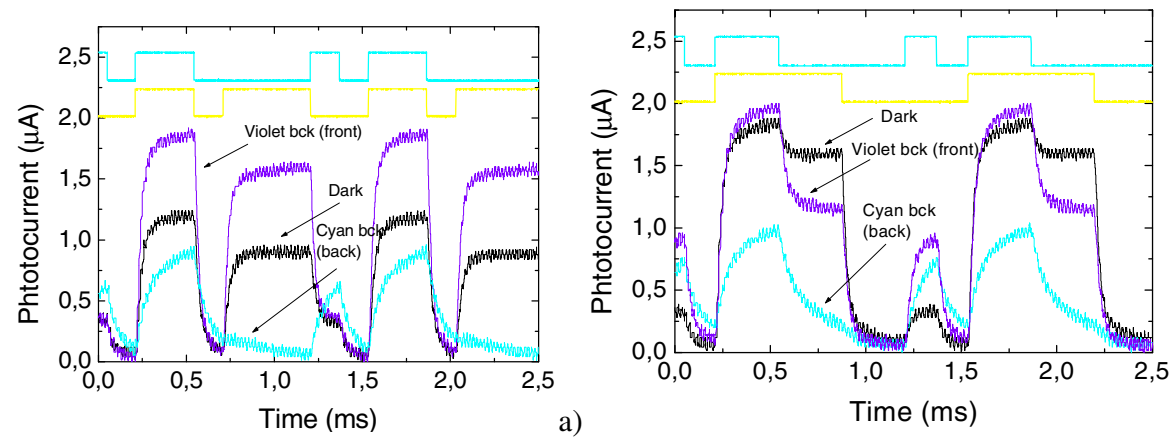

b)

Fig. 5. Output photocurrent signals without $\left(\Phi_{\mathrm{L}}=0\right)$ and with optical bias (violet light at $400 \mathrm{~nm}$ from the front side, and cyan light at $400 \mathrm{~nm}$ from the back side) of two different optical signals waveforms (shown at the top of the figure)

However, when the device is optically biased the output photocurrent changes enhancing the presence of each input fluorescent optical signal. In both sequences of Fig. 5 the shape of the photocurrent, measured by soaking the device with violet light by the front side, follows the shape of the yellow fluorescent signal). Whenever the yellow optical signal is $\mathrm{ON}$, the measured photocurrent is higher than its correspondent without optical bias. On the other hand, the use of cyan optical bias by the device back side results in an output signal that follows the cyan fluorescent emission, which allows the recognition of the presence of this fluorescent signal. Thus, the proper optical bias of the device through the choice of light wavelength and the device soaking light side results in the correct tuning of each fluorescence signal. These features allow immediate decoding of both yellow and cyan emitted signals. 


\section{$5 \quad$ Electrical Model}

Based on the experimental results and device configuration an optoelectronic model was developed [6]. The device was modeled by a two single-tuned stages circuit with two variable capacitors and interconnected phototransistors through a resistor (Fig. 6a). Two optical gate connections ascribed to the different light penetration depths across the front and back phototransistors were considered to allow independent yellow and cyan optical signals transmission.
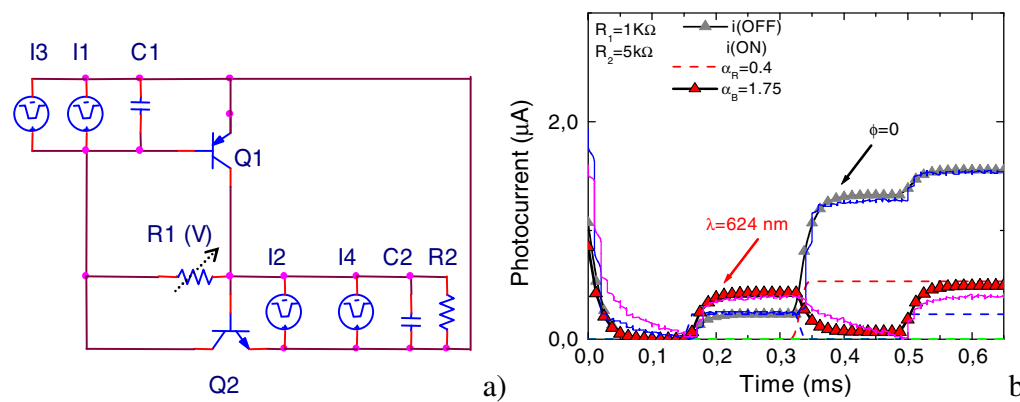

Fig. 6. a) Simplified ac equivalent electrical circuit of the device. b) Simulated photocurrent signal (symbols), input channels (dash lines) and experimental signal (solid lines): under reverse bias $(-8 \mathrm{~V})$ and red optical bias $(624 \mathrm{~nm})$.

The operation is based upon the following principle: the flow of current through the resistor connecting the two front and back transistor bases is proportional to the difference in the voltages across both capacitors (charge storage buckets). So, it uses a changing capacitance to control the power delivered to the load acting as a state variable filter circuit. In Figure $6 \mathrm{~b}$ the simulated current without and under red backgrounds is displayed (symbols) using the same test signal of Fig. 5a. The input channels are also displayed (lines). To simulate the red background, current sources intensities (input channels) were multiplied by the on/off ratio between the input channels with and without red optical bias. Good agreement between experimental and simulated data was observed. The four expected levels, under reversed bias, and their reduction under red irradiation, are clearly seen. Under red background the expected optical amplification of the cyan channel and the quenching of the yellow one were observed due to the effect of the active multiple-feedback filter when the back diode is light triggered.

\section{Conclusions}

We present a novel device semiconductor based on an a-SiC:H p-i-n/p-i-n heterostructures for the detection of optical signals near the cyan and yellow regions of the visible spectrum, which can be used for the detection of the emission signals used in the FRET technique. We used a commercial spectrofluorometer to measure 
the fluorescent signal emitted by recombinant plasmids from E. coli strain, which showed emission peaks at the cyan and yellow bands of the visible spectrum accordingly to the conformation changes suffered by the fluorescent proteins. Simultaneously different modulated optical signals were used to simulate the emission signals of the glucose nanosensors. The output emission spectrum was analyzed by reading out the photocurrent generated by the device under reverse bias and using different wavelengths of background light to soak the device from the front and back side. The influence of the fluorescent emission optical signals on the output photocurrent was also analyzed. Future work comprises the use of lower power intensities for the simulated emission signals in order to reach the same range of the ones produced during the FRET phenomenon. In a further stage tests with the emission fluorescence signals of the prepared recombinant plasmids from $E$. coli strain with the semiconductor device and the quantification of the glucose concentration from the changes in the measured spectra.

Acknowledgements. This work was supported by PTDC/EEA-ELC/111854/2009, PTDC/EEA-ELC/115577/2009 and PTDC/EEA-ELC/120539/2010.

\section{References}

1. Deuschle, K., Okumoto, S., Fehr, M., Looger, L.L., Kozhukh, L., Frommer, W.B.: Protein Sci. 14(9), 2304-2314 (2005)

2. Vieira, M.A., Vieira, M., Costa, J., Louro, P., Fernandes, M., Fantoni, A.: Sens. Transd. 9, 96-120 (2010)

3. Martins, S., Andrade, J., Karmali, A., Serralheiro, L.J.: Molecular Recognition 19, 340-347 (2006)

4. Louro, P., Vieira, M., Vieira, M.A., Fernandes, M., Fantoni, A., Francisco, C., Barata, M.: Physica E: Low-dimensional Systems and Nanostructures 41, 1082-1085 (2009)

5. Louro, P., Vieira, M., Fernandes, M., Costa, J., Vieira, M.A., Caeiro, J., Neves, N., Barata, M.: Phys. Status Solidi. C 7(3-4), 1188-1191 (2010)

6. Vieira, M., Fernandes, M., Louro, P., Fantoni, A., Vieira, M.A., Costa, J., Barata, M.: Phys. Status Solidi C 8(3), 1079-1082 (2011) 